\title{
STUDY OF INTERLEUKIN-33 IN IDIOPATHIC THROMBOCYTOPENIC PURPURA IN CHILDREN
}

\author{
Naglaa A. Khalifa Professor of Clinical Pathology, Faculty of Medicine - Zagazig University \\ Mervat Abdallah Hesham Professor of paediatrices, Faculty of Medicine - Zagazig University \\ Mervat Shaaban Lecturer of Clinical Pathology, Faculty of Medicine - Zagazig University \\ Samar Nageeb Ahmed M.B.B.CH Zagazig University
}

\section{Corresponding Author Samar Nageeb Ahmed 01066514669}

samarnageeb89@gmail.com

\section{INTRODUCTION}

mmune thrombocytopenia (ITP) is an immune-mediated hematologic disorder in which increased platelet destruction and
Background: Immune thrombocytopenia (ITP) is an immune-mediated hematologic disorder in which increased platelet destruction and decreased platelet production lead to thrombocytopenia and, thus, mucocutaneous bleeding. The interleukin-1 (IL-1) family is involved in many human diseases by regulating innate immunity and inflammation. As a new IL-1 family member found in 2005 , IL-33 and its receptor play important roles in inflammation, cancer, allergy and autoimmune diseases and are potential therapeutic targets. The aim of this work was to evaluate IL-33 among active ITP patients and remission in relation to control. Methods: This is a case control study This study was conducted in the Outpatient Clinic of Hematology Unit of Pediatric Department and Clinical Pathology Department at Zagazig university hospitals during the period from October 2017 to April 2018, on Twenty two ITP Patients during their regular follow-up visits and Twenty two age and sex-matched healthy children as a control group. Forty four subjects were included in this study; they were classified into two groups as follow: Control group and Patient group: Each group was subjected to the following: History, examination and Laboratory workup including IL-33.Results: There was no statistically significant difference between cases and control regarding sex and age.Purpura, ecchymosis and mucous membrane bleeding $(90.9 \%, 81.81 \%$ and $72.7 \%$ respectively) were the most common clinical data among ITP cases group. Less common splenomegaly and lymphadenopathy were $(4.54 \%$ and $.0 \%)$ respectively. There was no statistically significant difference between cases and control regarding HB, RBCs, WBCs and lymphocytes \%.Mean value of Platelets was significantly lower among cases than controls $(28.14,288.64$ respectively) $\mathrm{P}=.000$.Mean value of IL-33 was significantly lower among cases than controls (24.68, 47.18 respectively) $\mathrm{P}=.000$.There was statistically significant difference between acute ITP patients and chronic ITP patients regarding IL-33 (P value=.000). There was statistically significant difference between acute ITP patients and controls regarding IL-33 (P value $=.000$ ). There was no statistically significant difference between chronic ITP patients and controls regarding IL-33 (P value=.769). There were no statistically significant positive correlation between IL-33 and [age $(\mathrm{r}=.057, \mathrm{P}=.714), \mathrm{HB}(\mathrm{r}=.095, \mathrm{P}=.540)$ and RBCs $(.249, \mathrm{P}=.103$ ]. While There were statistically significant positive correlation between IL33 and [WBCs $(\mathrm{r}=.398, \mathrm{P}=.008)$, lymphocytes \% $(\mathrm{r}=.377, \mathrm{P}=.012)$ and Platelets $(\mathrm{r}=.537, \mathrm{P}=.000)$. Conclusion: This study concluded that, IL-33 may contribute to the pathogenesis of ITP. It could be a potential biomarker that diagnose and provide a new therapeutic target for ITP therapy.

Key words: interleukin-33-idiopathic thrombocytopenic purpurahematologic disorder.

decreased platelet production lead to thrombocytopenia and, thus, mucocutaneous bleeding. The pathophysiology of ITP has been extensively investigated. It is generally 
accepted that a complex multifactorial immune dysregulation, loss of immune tolerance, and generation of platelet autoantibodies account for the primary mechanism. Nevertheless, the underlying pathogenic events leading to the breakdown of immune tolerance in ITP remain elusive $^{(\mathbf{1})}$.In children, the disease sometimes follows a viral infection. In adults, it is more often a long-term (chronic) disease and can occur after a viral infection, with use of certain drugs, during pregnancy, or as part of an immune disorder. ITP affects women more often than men. It is more common in children than adults. In children, the disease affects boys and girls equally. ITP symptoms can include any of the following: Bleeding into the skin, often around the shins, causing a skin rash that looks like pinpoint red spots (petechial rash), Easy bruising and Nosebleed or bleeding in the mouth ${ }^{(2)}$

The interleukin-1 (IL-1) family is involved in many human diseases by regulating innate immunity and inflammation ${ }^{(3)}$.

As a new IL-1 family member found in 2005, IL-33 and its receptor play important roles in inflammation, cancer, allergy and autoimmune diseases and are potential therapeutic targets ${ }^{(4)}$

The value of IL-33 observed in patient with ITP correlated with disease activity, considering the role of IL-33 in regulating T cell immunity, studies on IL-33 in ITP would further improve our understanding of the pathogenesis of ITP ${ }^{(\mathbf{5}) \text {. }}$

The aim of this work was to evaluate IL-33 among active ITP patients and remission in relation to control.

\section{1- Subjects:}

\section{SUBJECTS AND METHODS}

This is a case control study This study was conducted in the Outpatient Clinic of Hematology Unit of Pediatric Department and Clinical Pathology Department at Zagazig university hospitals during the period from October 2017 to April 2018. Formal consent was obtained from all individual and the study protocol was approved by the Zagazig medical research ethical committee on Twenty two ITP Patients during their regular follow-up visits and Twenty two age and sexmatched healthy children as a control group.
Forty four subjects were included in this study; they were classified into two groups as follow:

A) Control group : It included 22 apparently healthy subjects. They were 16 males and 9 females. Their ages ranged from 3-9 years. They matched well with patients as regard age and sex .

B) Patient group: it include 22 ITP patients ,they were subgrouped into 2 groups:

1. Acute ITP group: It included 14 Acute ITP patients. They were 9 males and 5 females. Their ages ranged from 2-12 years.

2. Chronic ITP group: It included 8 chronic patients. They were 7 males and 1 females. Their ages ranged from 3-12 years.

Inclusion criteria include the following:

1.All children with ITP.

2.Age: from 2 to 14 years.

3.Sex: Both

Exclusion criteria:

1.Absence of informed consent.

2.Patients $<2$ years.

3.Patients $>14$ years.

\section{2-Methods:}

Each group was subjected to the following: A- Control group:

1. Routine laboratory investigations:

a. Complete blood count.

b. Liver and kidney function tests.

2. Measurement of serum IL-33.

B-Patient group:

1-Complete history:

- This included age, sex, patient complaint, onset of symptoms, duration, bleeding manifestations as petechaie, ecchymosis, mucous membrane bleeding, or bleeding from other orifices.

- History of preceding fever, viral infection, recent vaccination.

- History of drug intake to exclude drug induced thrombocytopenia

- History of blood transfusion for patients who had attacks of severe bleeding.

- History suggestive of collagen vascular disorder as arthritis and rash to exclude secondary causes of thrombocytopenia.

- History suggestive of viral hepatitis as jaundice, to exclude HCV and HBV.

- History of splenectomy for chronic ITP patients 


\section{Clinical examination:}

To detect

a- Organomegaly splenomegaly;

(hepatomegaly,

b- Lymphadenopathy

c- Petechiae or purpura

d- Unusual or easy bruising (haematoma)

e- Persistent bleeding symptoms from cuts or other injuries

f- Mucosal bleeding

g-Frequent or heavy nose bleeds (epistaxis)

h-Haemorrhage from any site (usually gingival or Menorrhagia in women)

i- Signs of secondary causes of immune thrombocytopenia such as malar rash of SLE or swollen tender joints

3. Laboratory workup:

a. Routine laboratory investigations:

1- Complete blood picture (CBC) .

2- Bone marrow aspiration .

3- Liver and kidney functions.

b. Special investigations: Measurement of IL-33 by using ELISA method.

\section{3-Statistical analysis:}

-The collected data were computerized and statistically analyzed using SPSS program (Statistical Package for Social Science) version 18.0.

1. Descriptive statistics: Data were summarized using the arithmetic mean, the standard deviation, median and range for numerical variables. The frequency, distribution and percentage were calculated for categorized variables.

2. Chi square test: chi square test was used to calculate difference between qualitative Variables

\section{Comparing numerical variables:}

A-Independent $T$ test: $\mathrm{T}$ test was used to calculate difference between quantitative variables in normally distributed data in two groups.

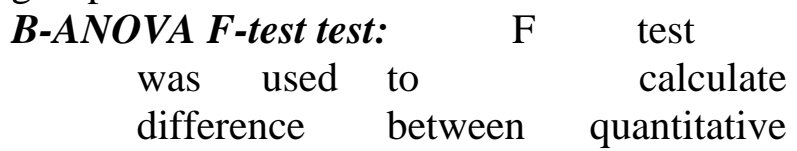

variables in more than two groups in normally distributed data.

\section{3. $\mathbf{r} \rightarrow$ Pearson's Product correlation coefficient:}

it evaluates the linear association between 2 quantitative variables ( one is the independent var.X, and the other is the dependent var., Y). value of " $r$ " ranges from -1 to 1

$0=$ no linear correlation

$1=$ perfect positive correlation

$-1=$ perfect negative correlation

Positive $=$ increase in the independent variable leads to increase in the dependent variable

Negative $=$ increase in the independent variable leads to decrease in the dependent variable

2. Level of significance: (P-value).

- $\mathrm{P}$ value of $>0.05$ indicates nonsignificant results.

- $\mathrm{P}$ value of $<0.05$ indicates a significant results.

\section{4-Results:}

There was no statistically significant difference between cases and control regarding sex and age. Table (1)

Purpura, ecchymosis and mucous membrane bleeding (90.9\%,81.81\% and $72.7 \%$ respectively) were the most common clinical data among ITP cases group. Less common splenomegaly and lymphadenopathy were $(4.54 \%$ and $.0 \%)$ respectively. Table (2)

There was no statistically significant difference between cases and control regarding HB, RBCs, WBCs and lymphocytes \%.Mean value of Platelets was significantly lower among cases than controls (28.14, 288.64 respectively) $\mathrm{P}=.000$ Table (3)

Mean value of IL-33 was significantly lower among cases than controls (24.68, 47.18 respectively) $\mathrm{P}=.000$ Table (4)

There was statistically significant difference between acute ITP patients and chronic ITP patients regarding IL-33 (P value=.000). There was statistically significant difference between acute ITP patients and controls regarding IL-33 (P value $=.000)$. There was no statistically significant difference between chronic ITP patients and controls regarding IL-33 (P value=.769). Table (5) There were no statistically significant positive correlation between IL-33 and [age $(r=.057$, $\mathrm{P}=.714)$, HB ( $\mathrm{r}=.095, \mathrm{P}=.540)$ and RBCs (.249, $\mathrm{P}=.103]$. While There were statistically significant positive correlation between IL-33 and [WBCs ( $\mathrm{r}=.398, \mathrm{P}=.008)$, lymphocytes \% $(\mathrm{r}=.377, \mathrm{P}=.012)$ and Platelets $(\mathrm{r}=.537, \mathrm{P}=.000)]$ Table (6) 
Table 1 Demographic characteristics of the studied group

\begin{tabular}{|c|c|c|c|c|c|c|}
\hline & & & & controls & $\mathbf{X} 2$ & P. value \\
\hline \multirow[t]{4}{*}{ Sex } & \multirow[t]{2}{*}{ Male } & No. & 16 & 16 & \multirow[t]{4}{*}{.000} & \multirow[t]{4}{*}{1.00} \\
\hline & & $\%$ & $72.7 \%$ & $72.7 \%$ & & \\
\hline & \multirow[t]{2}{*}{ Female } & No. & 6 & 6 & & \\
\hline & & $\%$ & $27.3 \%$ & $27.3 \%$ & & \\
\hline \multirow[t]{2}{*}{ Age (years) } & Range & \multicolumn{2}{|c|}{$2-12$} & $3-9$ & \multirow[t]{2}{*}{.418} & \multirow[t]{2}{*}{.678} \\
\hline & Mean \pm SD & \multicolumn{2}{|c|}{$5.86 \pm 3.04$} & $5.55 \pm 1.87$ & & \\
\hline
\end{tabular}

Table 2 Clinical data of the cases group.

\begin{tabular}{|c|c|c|}
\hline Variable & \multicolumn{2}{|c|}{ ITP group } \\
\cline { 2 - 3 } & No. & \% \\
\hline Purpura & 20 & $90.9 \%$ \\
\hline Mucous membrane bleeding & 16 & $72.7 \%$ \\
\hline Ecchymosis & & \\
\hline Splenomegaly & 18 & $81.81 \%$ \\
\hline Lymphadenopathy & 1 & $4.54 \%$ \\
\hline & & $.0 \%$ \\
\hline
\end{tabular}

Table 3 Complete blood count of the studied groups.

\begin{tabular}{|c|c|c|c|c|c|}
\hline & & cases & controls & t.test & P. value \\
\hline \multirow[t]{2}{*}{ HB (g/dl) } & Range & $8.4-13.8$ & $10-12.6$ & \multirow[t]{2}{*}{.925} & \multirow[t]{2}{*}{.360} \\
\hline & Mean \pm SD & $11.35 \pm 1.48$ & $11.03 \pm .697$ & & \\
\hline \multirow[t]{2}{*}{$\operatorname{RBCs}\left(\times 10^{6} / \mathrm{uL}\right)$} & Range & $3.9-5.1$ & $3.8-5.1$ & \multirow[t]{2}{*}{1.18} & \multirow[t]{2}{*}{.244} \\
\hline & Mean \pm SD & $4.49 \pm .342$ & $4.35 \pm .441$ & & \\
\hline \multirow[t]{2}{*}{ WBCs $\left(x^{2} 10^{3} / \mathrm{uL}\right)$} & Range & $4 . \overline{-16}$ & $8.8-14$ & \multirow[t]{2}{*}{.857} & \multirow[t]{2}{*}{.396} \\
\hline & Mean+SD & $10.61 \pm 3.30$ & $11.30 \pm 1.88$ & & \\
\hline \multirow[t]{2}{*}{ lymphocytes \% } & Range & $18-60$ & $45-60$ & \multirow[t]{2}{*}{1.70} & \multirow[t]{2}{*}{.095} \\
\hline & Mean \pm SD & $45.64+14.58$ & $51.27 \pm 5.14$ & & \\
\hline \multirow[t]{2}{*}{ Platelets $\left(\times 10^{3} / \mathrm{uL}\right)$} & Range & $5-64$ & $180-445$ & \multirow[t]{2}{*}{13.42} & \multirow[t]{2}{*}{.000} \\
\hline & Mean \pm SD & $28.14 \pm 22.12$ & $288.64+88.26$ & & \\
\hline
\end{tabular}

Table 4 Comparison between cases and controls regarding IL-33.

\begin{tabular}{|c|c|c|c|l|l|}
\hline \multicolumn{2}{|c|}{} & cases & controls & t.test & P. value \\
\hline \multirow{2}{*}{ IL-33 (ng/L) } & Range & $5-58$ & $36-67$ & 5.13 & .000 \\
\cline { 2 - 6 } & Mean \pm SD & $24.68 \pm 17.76$ & $47.18 \pm 10.32$ & & \\
\hline
\end{tabular}


Table 5 Comparison between acute ITP patients, chronic ITP patients and controls regarding IL-33.

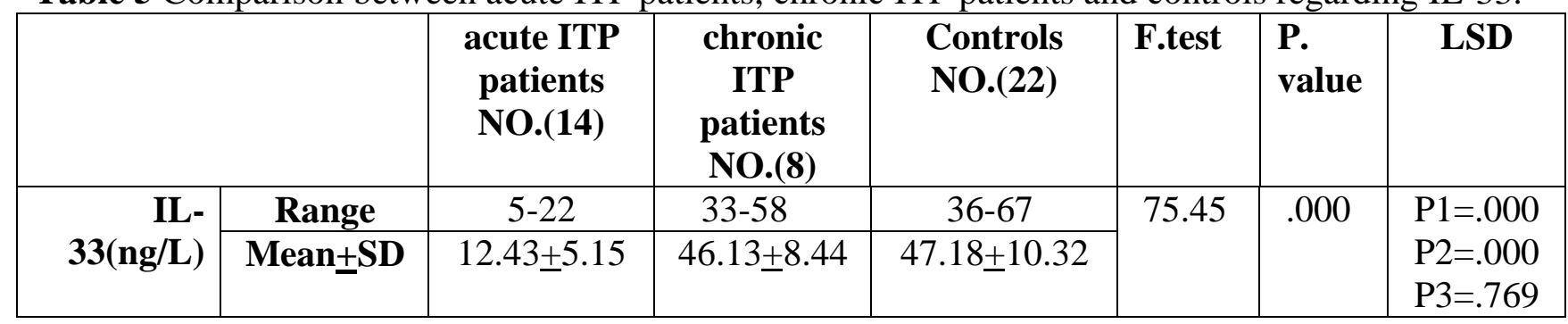

Table 6 Correlation between IL-33 and other variables.

\begin{tabular}{|c|c|c|}
\hline \multirow{2}{*}{ IL-33 } & \multicolumn{2}{|c|}{ Pearson's correlation } \\
\cline { 2 - 3 } & r & p \\
\hline Age (years) & .057 & .714 \\
\hline Hb & .095 & .540 \\
\hline RBCs & .249 & .103 \\
\hline WBCs & .398 & .008 \\
\hline lymphocytes \% & .377 & .012 \\
\hline Platelets & .537 & .000 \\
\hline
\end{tabular}

\section{DISCUSSION}

This study was carried out on 44 subjects (22 cases and 22 controls).Cases are subgrouped into 2 groups (Acute, Chronic).Acute ITP group included 14 patients, they were 9 males and 5 females. Their age ranged from 212.Chronic ITP group included 8 patients; they were 7 males and 1 female. Their age ranged from 3-12 years.

This agreed with ${ }^{(6)}$ who aimed to assess the clinical role of a cytokine in patients with ITP and correlate its levels with different stages of the disease. A total of 20 patients with ITP at the onset stage, 40 patients with chronic ITP, and 30 healthy matched controls were enrolled in this study. He found that, $15.6 \%$ were males and $84.4 \%$ were females, showing a female predominance, with age range of 18-50 years with mean of 31.8 years, which was in agreement with the results of ${ }^{(\mathbf{7 , 8 , 9})}$ All these studies were performed on patients with ITP and showed that ITP among adults affects females more than males, with no specific age predominance. On comparing our three groups, we found no statistically significant difference between them.

On the contrary, ${ }^{(10)}$ showed that the incidence of ITP is predominant in males in childhood.

In current study clinical data in ITP group was evaluated, purpura was found in 20 patients (90.9\%), followed by Ecchymosis which was found in 18 patients $(81.81 \%)$. Mucous membrane bleeding was found in 16 patients (72.7\%), Splenomegaly was found in one patient $(4.54 \%)$, while lymphadenopathy was not found in any patients.

Since platelets play a pivotal role in primary hemostasis, quantitative and/or qualitative abnormalities may present with bleeding symptoms. In patients with ITP, the most common bleeding symptoms are purpura, mucocutaneous bleeding and prolonged bleeding after minor injury. Rarely, patients may present with bleeding in vital organs or excessive bleeding after hemostatic challenge ${ }^{(11)}$

This agreed with ${ }^{(\mathbf{1 2})}$ found that, that the most common symptoms were skin rash $(93.5 \%)$ and epistaxis $(41.3 \%)$, which was significantly more among male children. The most common signs were ecchymosis $(87 \%)$, purpura $(76.1 \%)$, petechial rash $(60.9 \%)$, bleeding in mucous membranes $(58.7 \%)$ and pallor $(32.6 \%)$. Splenomegaly was present in $6.5 \%$ of patients.

This study showed that, mean value of platelets was significantly lower among cases than controls .

Platelets count were severely decreased in ITP patients than controls due to presence of platelet autoantibodies directed against specific platelet glycoproteins, such antibodies can be 
detected in $40 \%$ to $80 \%$ of patients with clinical ITP. However, absence of detectable antibodies does not rule out a diagnosis of ITP, since many patients without detectable antibodies respond to traditional ITP therapies and antibodies may occur in patients with liver disease and other conditions without clinical $\operatorname{ITP}^{(13)}$

This agreed with ${ }^{(6)}$ who aimed to assess the clinical role of a cytokine in patients with ITP and correlate its levels with different stages of the disease. A total of 20 patients with ITP at the onset stage, 40 patients with chronic ITP, and 30 healthy matched controls were enrolled in this study. He revealed a highly statistically significant difference between the groups regarding platelets count, with lower counts in patients with acute ITP than those with chronic $\operatorname{ITP}(\mathrm{p}<0.001)$.

This may be attributed to platelet destruction arises from antibodies generated during the immune response to a viral or bacterial infection in acute ITP that cross-react with platelet antigens, also other mediators of the immune response arising from infection may play an additional role in suppressing platelet production ${ }^{(\mathbf{1 4})}$

Serum IL-33 was measured by using ELISA technique because it is one of the most widely used, best validated, and most sensitive immunoassays available. ELISA is also quick, easy, less expensive, and it is a very popular choice for the evaluation of various targets in clinical laboratories and biomedical research since it allows processing of even larger numbers of samples. Additional advantages of ELISA include the fact that results are highly quantitative and generally reproducible. ${ }^{(15)}$

This study showed that, mean value of IL-33 was significantly lower among cases than controls $(24.68,47.18$ respectively).There was statistically decrease of IL-33 among acute ITP children than chronic ITP children (P value $=.000)$. There was statistically decrease of IL-33 among acute ITP children than controls ( $\mathrm{P}$ value $=.000$ ). There was no statistically significant difference between chronic ITP children and controls regarding IL-33 (P value $=.769$ ).
This agreed with ${ }^{(\mathbf{1 5})}$ who aimed to measure the expression of IL-33 in active immune thrombocytopenia (ITP) compared to controlsThirty-two ITP patients measured in plasma using ELISA and compared with 27 age- and sex-matched healthy controls.IL-33 in the ITP patients with active disease were significantly lower than those of the healthy controls. Significantly lower IL-33 and higher sST2 plasma levels were found in the active ITP patients compared with the ITP patients in .However, no significant differences in the IL33 and sST2 plasma levels were found between the ITP patients in remission and healthy controls. Interestingly, the expression of IL-33 was unchanged in the patients compared with the controls. There was no significant difference between the patients in remission and the control group.

A number of studies suggest that a generalized dysregulation in immune regulatory networks may be present in ITP as in other autoimmune diseases. (16,17)

This study showed that, there were no statistically significant positive correlation between IL-33 and HB and RBCs. While There were statistically significant positive correlation between IL-33 ,WBCs, lymphocytes \% and Platelets .

(15) made correlations between the plasma IL33 and platelet counts were analyzed in the active ITP patients. The data demonstrated no correlation between the IL-33 levels and platelet counts. No correlation between sST2 concentrations and platelet counts was found in the ITP patients with active disease. The plasma levels of IL-33 in the ITP patients with active disease were significantly decreased, and the plasma levels of sST2 were significantly increased compared with the normal controls. The ratio of sST2/IL-33 in the patients with active disease was significantly increased compared with the normal and remission groups ( $\mathrm{P}$ b 0.01). However, there was no significant difference between the patients in remission and normal controls. ${ }^{(15)}$

\section{CONCLUSION}

This study concluded that, IL-33 may contribute to the pathogenesis of ITP. It could be a potential biomarker that diagnose and 
provide a new therapeutic target for ITP therapy.

\section{REFERENCES}

1. Stasi R, Cooper N, Del Poeta G, (2008) Analysis of regulatory $\mathrm{T}$-cell changes in patients with idiopathic thrombocytopenic purpura receiving $\mathrm{B}$ cell-depleting therapy with rituximab. Blood 112(8):1147-1150.

2. Arnold DM, Patriquin C, Toltl LJ, Nazi I, Smith J, Kelton J. (2013) Diseases of platelet number: immune thrombocytopenia, neonatal alloimmune thrombocytopenia, and posttransfusion purpura. In: Hoffman R, Benz EJ, Silberstein LE, Heslop HE, Weitz JI, Anastasi J, eds. Hematology: Basic Principles and Practice. 6th ed. Philadelphia, PA: Elsevier Saunders; 2013:chap 133.

3. Garlanda C., C.A. Dinarello, A. Mantovani, (2013) The interleukin-1 family: back to the future, GImmunity 39 (6) 1003-1018.

4. Pei C., M. Barbour, K.J. Fairlie-Clarke, D. Allan, R. Mu, H.R. Jiang, (2014) Emerging role of interleukin-33 in autoimmune diseases, Immunology 141 (1) 9-17.

5. Liu X., Y. Hou, J. Peng, (2013) Advances in immunopathogenesis of adult immune thrombocytopenia, Front. Med. 7 (4) 418-424.

6. Hamed H, Moussa M, Fathey H, Tolba H. (2018) Role of measurement of interleukin 10 in idiopathic (immune) thrombocytopenic purpura. Egypt J Haematol [serial online] 2017 [cited 2018 Apr 20];42:148-54. Available from:

7. Zhang F., J.T. Tossberg, C.F. Spurlock, S.Y. Yao, T.M. Aune, S. (2014) Sriram, Expression of IL-33 and its epigenetic regulation in Multiple Sclerosis, Ann. Clin. Transl. Neurol. 1 (5) 307-318.

8. Talaat R.M., A.M. Elmaghraby, S.S. Barakat, M. El-Shahat, (2014) Alterations in immune cell subsets and their cytokine secretion profile in childhood idiopathic thrombocytopenic purpura (ITP), Clin. Exp. Immunol. 176 (2) 291-300.
9. Elsalakawy WA, Ali MA, Hegazy MG (2014) Value of vanin-1 assessment in adult patients with primary immune thrombocytopenia. Platelets 2014; 25:86-92.

10. Arandi N, Mirshafiey A, Jeddi-Tehrani M, Shaghaghi M, Sadeghi B, Abolhassani H, et al. (2014) Alteration in frequency and function of CD4(+)CD25(+)FOXP3 (+) regulatory T cells in patients with immune thrombocytopenic purpura. Iran J Allergy Asthma Immunol;13:85-92

11. Rodeghiero F, Stasi R, Gernsheimer T, (2009) Standardization of terminology, definitions and outcome criteria in immune thrombocytopenic purpura of adults and children: report from an international working group. Blood 113(11):2386-2393.

12. AL-SUHEEL A, SHATI A, SULTAN A. (2014) Immune Thrombocytopenia among Children Living at a High Altitude Region: A Hospital-Based Retrospective Study, Med. J. Cairo Univ., Vol. 82, No. 2, December: 87-93, 2014 www.medicaljournalofcairouniversity.net

13. McMillan, R. (2009). Update on chronic immune thrombocytopenic purpura (ITP). Journal of Hematology \& Oncology, 2(Suppl 1), A5.

14. Cines, D. B., Bussel, J. B., Liebman, H. A., \& Luning Prak, E. T. (2009). The ITP syndrome: pathogenic and clinical diversity. Blood, 113(26), 6511-6521. http://doi.org/10.1182/blood-2009-01-129155

15. Li PP, Zhang XM, Yuan D, Liu X, Li Y, Shan NN. (2015): Decreased expression of IL-33 in immune thrombocytopenia. Int Immunopharmacol 2015; 28: 420-424.

16. Sakakura M, Wada H, Tawara I, (2007): Reduced $\mathrm{Cd} 4+\mathrm{Cd} 25+\mathrm{T}$ cells in patients with idiopathic thrombocytopenic purpura. Thromb.Res. 120:187-193.

17. Li M, Li Y, Liu X, Gao X, Wang Y. (2012) IL33 blockade suppresses the development of experimental autoimmune encephalomyelitis in $\mathrm{C} 57 \mathrm{BL} / 6$ mice. Journal of Neuroimmunology.;247(1-2):25-31.

How to cite this article: Khalifa NA, Hesham MA, Shaaban M, Ahmed SN. Study of Interleukin33 in Idiopathic Thrombocytopenic Purpura in Children . ZUMJ 2019; 25 (1); 164-170. 